\title{
Study of crystallite size and strain as a function of morphological evolution in zinc oxide powder obtained from hydroxycarbonate precursor
}

\author{
Fernando Aparecido Sigoli, Carlos de Oliveira Paiva-Santos, Miguel Jafelicci, Jr., \\ and Marian Rosaly Davolos ${ }^{\text {a) }}$ \\ Instituto de Química, Universidade Estadual Paulista-UNESP, P.O. Box 355, 14801-970, Araraquara, SP, \\ Brazil
}

(Received 12 February 2000; accepted 3 April 2001)

\begin{abstract}
In this work, zinc oxide samples were obtained from hydroxycarbonate by thermal decomposition at $300^{\circ} \mathrm{C}$. Zinc hydroxycarbonate samples were produced by homogeneous precipitation over different periods of time. The method used to obtain zinc oxide produces different morphologies as a function of the precursor precipitation time. Among the obtained particle shapes were porous spherical aggregates, spherulitic needle aggregates, and single acicular particles. This work investigated spherulitic needle-aggregate formation and the correlation among morphology, domain size, and microstrain. Transmission electron microscopy data revealed that the acicular particles that form the spherulitic needle aggregates consist of nanometer crystallites. Apparent crystallite size and microstrain in the directions perpendicular to $(h 00),(h 0 l),(h k 0)$, and $(00 l)$ planes were invariable as a function of precursor precipitation time. From the results, it was possible to conclude that the precursor precipitation period directly influenced the morphology of the zinc oxide but did not influence average crystallite size and microstrain for $\mathrm{ZnO}$ samples. Therefore, using this route, it was possible to prepare zinc oxide with different morphologies without microstructural alterations. (c) 2001 International Centre for Diffraction Data. [DOI: 10.1154/1.127606]
\end{abstract}

Key words: zinc oxide, crystallite size, strain, morphology

\section{INTRODUCTION}

Precursors with low thermal stability, e.g., oxalates, hydroxynitrates, and hydroxycarbonates, can be transformed to oxides by endothermic thermal decomposition at about $300^{\circ} \mathrm{C}$, generally as finely divided solid particles (Auffrédic et al., 1995). The importance of endothermic decomposition in the formation of solids with high surface area is recognized in several fields of material science, e.g., sintering, catalysts, pigments, and reactivity of solids (Langford et al., 1993). It is commonly accepted that morphology and microstructure of the particles in these solids depend on the nature of the precursor and also on the experimental parameters chosen for carrying out the solid state decomposition of precursors (Auffrédic et al., 1995). In recent years a large number of methods have been developed to prepare particles of different compositions, but with uniform shapes and narrow size distributions in the micron and submicron range (Matjévic, 1985). The most convenient method for preparation of such products is based on the control of chemical processes taking place in solutions (Haruta et al., 1986).

Zinc oxide is one of the most promising materials among $\mathrm{A}^{\mathrm{II}} \mathrm{B}^{\mathrm{VI}}$ wide-band semiconductors (Georgobiami et al., 1993). The utilization of zinc oxide as a semiconductor is limited by the difficulties in obtaining samples with the proper electrical and optical properties (Georgobiami et al., 1993). Energy absorption in this semiconductor occurs by exciting electrons to the empty conduction band leaving holes in the completely filled valence band. Recombination usually occurs close to or in electronic defects of the crystal lattice (Blasse and Grabmaier, 1994). Such defects normally depend on morphology, structure, particle size, composition,

${ }^{a}$ Electronic mail: davolos@iq.unesp.br and crystallinity (Kouldelka and Horák, 1994), which play an important role in luminescent devices and pigment technologies (Vanheusden et al., 1997). ZnO is industrially produced in large quantities by the oxidation of zinc vapor, resulting in various morphologies, and also by thermal decomposition of a topotactic precursor such as zinc oxalate or zinc hydroxynitrate (Collins and Taylor, 1992). The zinc hydroxycarbonate precursor allows zinc oxide particles to be prepared with controlled size and shape. It is prepared by aging aqueous zinc salt solutions in the presence of urea at a controlled temperature (Castellano and Matijévic, 1989). Spherulitic needle aggregates of zinc oxide were obtained from a chloride medium and large porous spherical aggregates from a sulfate medium using this method (Zampronio et al., 1995).

Morphology, composition, crystal structure, crystallite size, and crystallinity depend fundamentally on the precursors and on experimental parameters used during the solidstate decomposition (Louër et al., 1983). Techniques used for the investigation of these characteristics include X-ray diffractometry, scanning and transmission electron microscopies, and specific surface area. X-ray diffraction line broadening depends on the crystallite size and can be used as a nondestructive method to obtain information on the crystallite size and shape, and also to evaluate the existence of structural imperfections. Zinc oxide is an interesting case on which to perform $\mathrm{X}$-ray diffraction line broadening analysis because it presents a rich X-ray diffractogram with various orders of reflection, which allow crystallite shape and average size analysis. Some zinc oxide properties, such as electrical and optical, can be correlated with microstructural characteristics that depend on zinc oxide precursor precipitation and decomposition. 


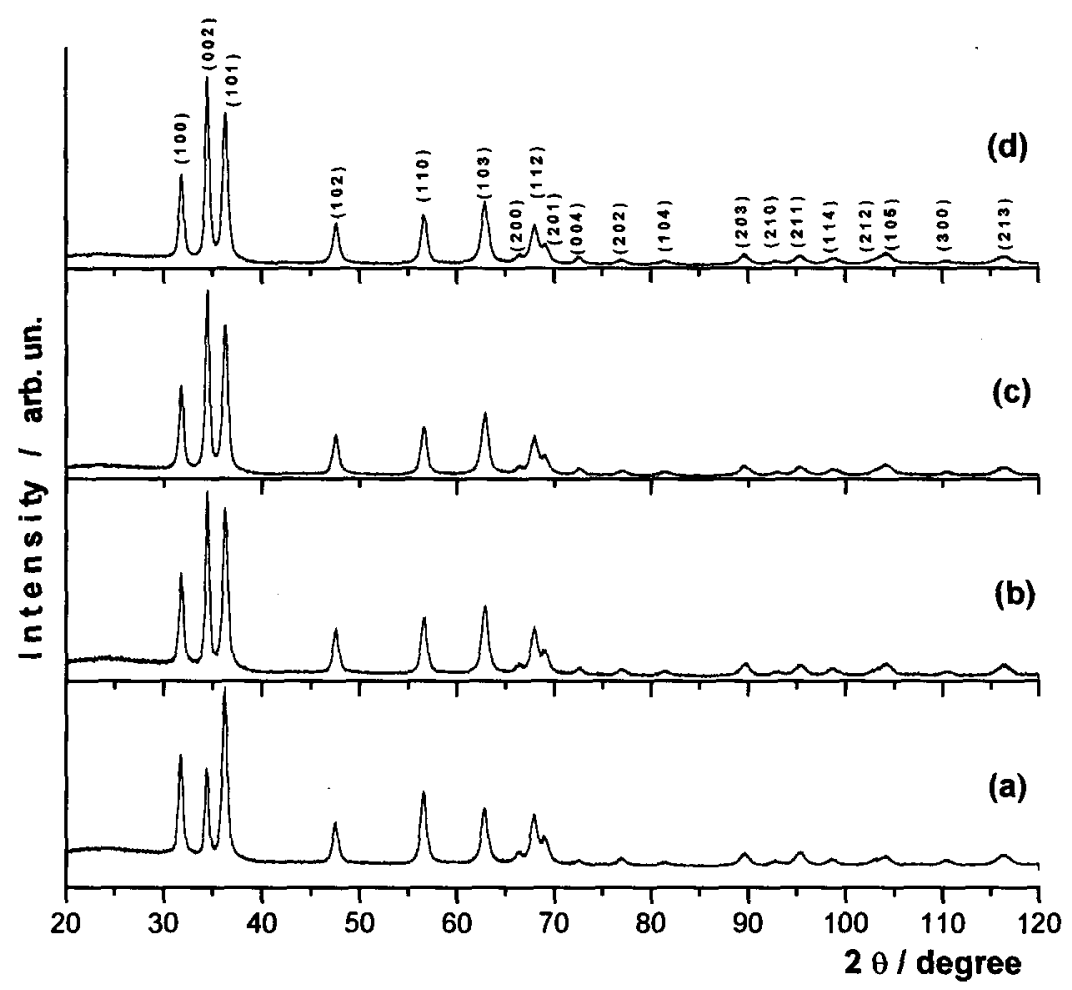

Figure 1. X-ray diffractogram of zinc oxide samples obtained at different precursor precipitation times: (a) $\mathrm{ZnO}-30$, (b) $\mathrm{ZnO}-180$, (c) $\mathrm{ZnO}-250$, (d) $\mathrm{ZnO}-440$.

(b)

In a recent work, we have obtained zinc oxide samples containing porous spherical, spherulitic needle aggregates or needle particles, depending on the precipitation time, from a zinc chloride medium (Sigoli et al., 1997). The present work investigated spherulitic needle-aggregate formation and the correlation among morphology, domain size, and microstrain in the $\mathrm{ZnO}$ obtained from endothermic decomposition of zinc hydroxide carbonate.

\section{EXPERIMENT}

\section{A. Sample preparation}

Zinc oxide samples, $\mathrm{ZnO}-30, \mathrm{ZnO}-180, \mathrm{ZnO}-250$, and $\mathrm{ZnO}-440$, were obtained from thermal decomposition carried out in an oven (EDG FIVE-4) at $300^{\circ} \mathrm{C}$ for $240 \mathrm{~min}$ from zinc hydroxycarbonate, $\mathrm{Zn}_{5}\left(\mathrm{CO}_{3}\right)_{2}(\mathrm{OH})_{6}$. The latter was precipitated from homogeneous precipitation at different times: $30,180,250$, and $440 \mathrm{~min}$, respectively, as described by Sigoli et al. (1997).

\section{B. Powder characterization}

Zinc oxide samples were characterized by a transmission electron microscopy (ZEISS EM 902), specific surface using the BET method (SCIENTIFIC INSTRUMENTS CG 2000) and X-ray diffraction (SIEMENS D5000) using a $\mathrm{Cu}$ radiation source and a graphite monochromator. The X-ray diffraction patterns for $\mathrm{ZnO}$ samples were obtained over the

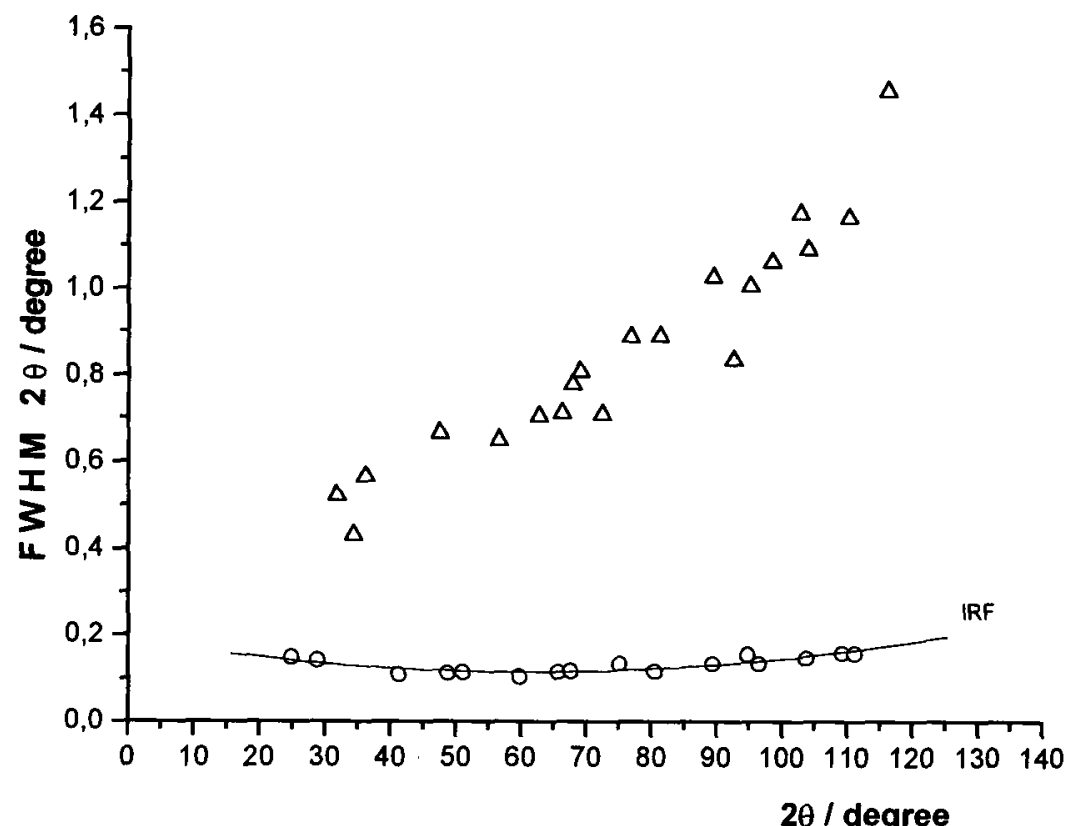

Figure 2. FWHM values as function of $2 \theta$. $(O) \mathrm{BaF}_{2}$, internal reference factor (IRF), $(\triangle) \mathrm{ZnO}-30$ obtained by thermal decomposition of precursor precipitated at 30 min. 
angular range 4 to $120^{\circ} 2 \theta$, with a step size of $0.02^{\circ} 2 \theta$, and counting time of $10 \mathrm{~s} \mathrm{step}^{-1}$.

\section{Microstructural analysis}

Zinc oxide X-ray diffraction data (Figure 1) were used to investigate the mean crystallite size and the microstrain. Instrumental broadening corrections were performed using a standard of $\mathrm{BaF}_{2}$ annealed for $36 \mathrm{~h}$ at $900^{\circ} \mathrm{C}$. The standard $\mathrm{X}$-ray diffraction XRD pattern was obtained using the same conditions as for the $\mathrm{ZnO}$ samples. The full width at halfmaximum (FWHM) for peaks of all $\mathrm{ZnO}$ samples and the standard was obtained by using the program PRO-FIT (Toraya, 1990). The FWHM of the $\mathrm{BaF}_{2}$ standard together with one of the zinc oxide samples is plotted in Figure 2, showing the good quality of the standard used. For microstrain and apparent crystallite size investigations the integral breadth $\left(\beta_{f}\right.$ $=$ area/maximum intensity), was obtained considering that the line broadening for the samples, $h(x)$, and the instrumental broadening, $g(x)$, are expressed by the Voigt function. The use of this function can be justified by $(\varphi$ $=2 \mathrm{FWHM} / \beta$ ) whose values must be between the Lorentzian $(\varphi=0.634)$ and Gaussian $(\varphi=0.939)$ limits (Langford et al., 1993). By assuming the line profiles as a Voigt function, the integral breadth, $\left(\boldsymbol{\beta}_{f}\right)$, and instrumental corrections can be obtained by the method proposed by Langford et al. (1988). The apparent crystallite sizes in the directions perpendicular to the (100), (002), (101), (102), (110), (103)
TABLE I. Experimental $\left(S_{\mathrm{BET}}\right)$ and calculated $\left(S_{\mathrm{XRD}}\right)$ surface areas and agglomeration degree $(1 / F)$ for $\mathrm{ZnO}$ samples.

\begin{tabular}{lccccc}
\hline \hline & $\left\langle H_{v}\right\rangle(\mathrm{nm})$ & $\left\langle D_{v}\right\rangle(\mathrm{nm})$ & $S_{\text {XRD }}\left(\mathrm{m}^{2} \mathrm{~g}^{-1}\right)$ & $S_{\text {BET }}\left(\mathrm{m}^{2} \mathrm{~g}^{-1}\right)$ & $1 / F$ \\
\hline ZnO-30 & $23(1.2)$ & $14.14(0.7)$ & 65.20 & 31.55 & 0.48 \\
ZnO-180 & $20(1.0)$ & $14.14(0.8)$ & 67.50 & 28.28 & 0.41 \\
ZnO-250 & $18(1.1)$ & $14.14(0.7)$ & 69.45 & 30.87 & 0.44 \\
ZnO-440 & $18(0.9)$ & $14.14(0.7)$ & 69.45 & 30.62 & 0.44 \\
\hline \hline
\end{tabular}

planes and the lattice strain were obtained from the singleline method (Langford et al., 1988) and are listed in Table I. In this method it is normally assumed that $\beta_{L f}$ is due only to size effects and $\beta_{G f}$ is attributed to strain. Since this approach is applied to data for each line separately, information on crystallite shape and strain can be obtained, but the results will clearly be influenced by this assumption (Langford 1992). Using the single-line method the crystallite size was obtained by Eq. (1), the strain, $\eta$, was calculated by expression (2), and the rms strain, $e$, was obtained from Eq. (3):

$$
\begin{gathered}
\beta_{L f}=1 / \epsilon, \\
\beta_{G f}=\eta d^{*} / 2, \\
e=\eta /(2 \sqrt{(2 \pi)}) \cong \eta / 5 .
\end{gathered}
$$

The specific surface areas obtained from the BET method, $S_{\mathrm{BET}}$, and those calculated from the crystallite
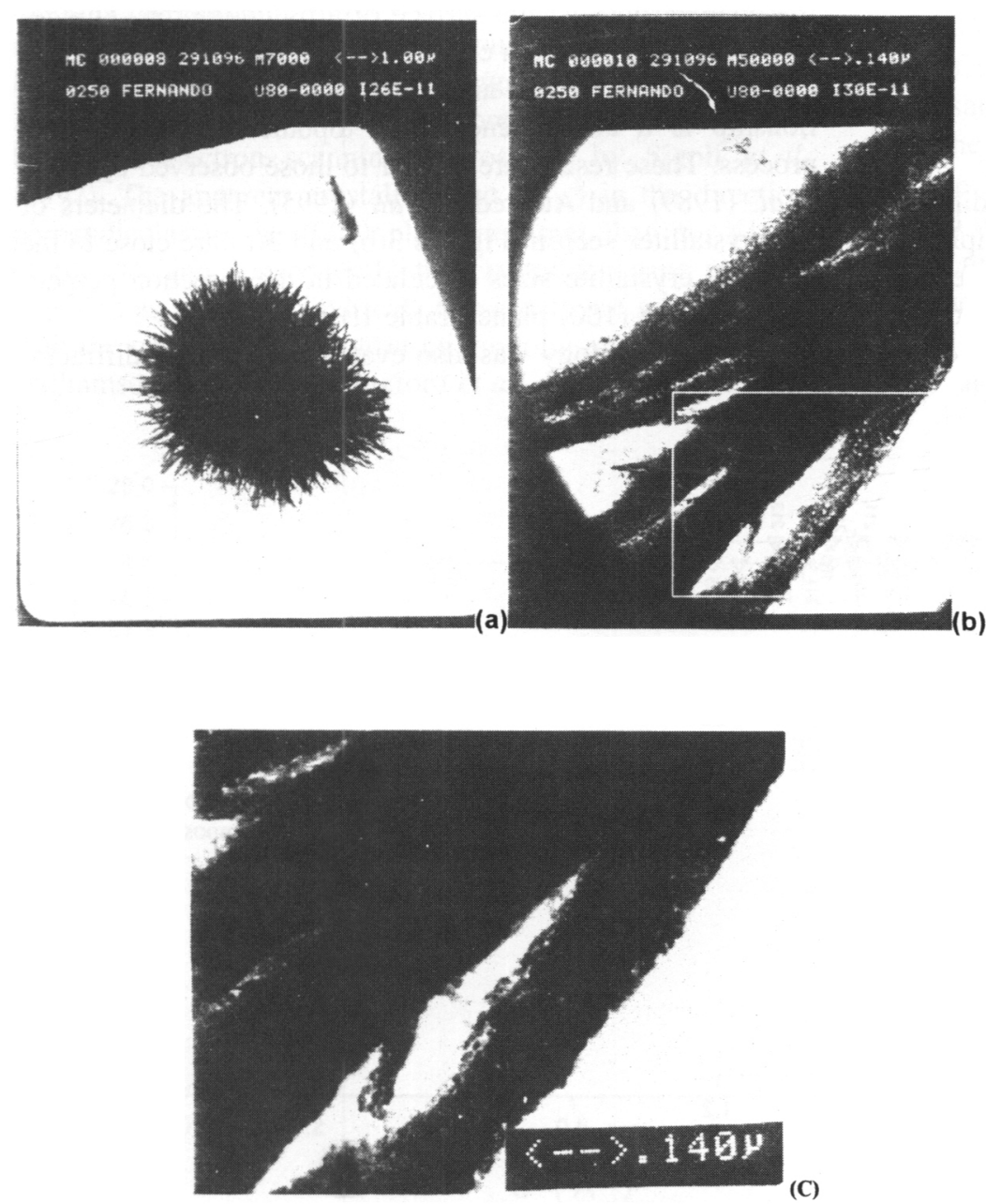

Figure 3. TEM micrographs of $\mathrm{ZnO}$ obtained by thermal decomposition of precursor precipitated at $250 \mathrm{~min}$ (a) spherulitic aggregates $7000 \times$, (b) acicular particles of spherulitic aggregates $50000 \times$, and (c) magnification of selected area showing subparticle details. 
TABLE II. Calculated apparent size $\left(\epsilon_{h k l}\right), \eta$, and rms strain from single-line method.

\begin{tabular}{|c|c|c|c|c|c|c|c|c|c|c|c|c|}
\hline \multirow[b]{2}{*}{$h k l$} & \multicolumn{3}{|c|}{$\mathrm{ZnO}-30$} & \multicolumn{3}{|c|}{$\mathrm{ZnO}-180$} & \multicolumn{3}{|c|}{$\mathrm{ZnO}-250$} & \multicolumn{3}{|c|}{$\mathrm{ZnO}-440$} \\
\hline & $\epsilon(\AA)$ & $\begin{array}{c}\eta \\
\left(\times 10^{2}\right)\end{array}$ & $\begin{array}{c}\text { Strain } \\
\left(\times 10^{3}\right)\end{array}$ & $\epsilon(\AA)$ & $\begin{array}{c}\eta \\
\left(\times 10^{2}\right)\end{array}$ & $\begin{array}{c}\text { Strain } \\
\left(\times 10^{3}\right)\end{array}$ & $\epsilon(\AA)$ & $\begin{array}{c}\eta \\
\left(\times 10^{2}\right)\end{array}$ & $\begin{array}{c}\text { Strain } \\
\left(\times 10^{3}\right)\end{array}$ & $\epsilon(\AA)$ & $\begin{array}{c}\eta \\
\left(\times 10^{2}\right)\end{array}$ & $\begin{array}{c}\text { Strain } \\
\left(\times 10^{3}\right)\end{array}$ \\
\hline 100 & 168 & 2.4 & 4.8 & 140 & 1.9 & 3.8 & 141 & 2.1 & 4.2 & 155 & 2.3 & 4.5 \\
\hline 002 & 229 & 1.6 & 3.3 & 198 & 1.6 & 3.2 & 177 & 1.8 & 3.6 & 183 & 1.8 & 3.6 \\
\hline 101 & 154 & 2.3 & 4.7 & 133 & 2.1 & 4.3 & 123 & 2.1 & 4.3 & 127 & 2.2 & 4.4 \\
\hline 102 & 92 & 1.7 & 3.3 & 99 & 1.8 & 3.5 & 83 & 1.7 & 3.4 & 86 & 1.8 & 3.6 \\
\hline 110 & 122 & 1.7 & 3.4 & 122 & 1.7 & 3.5 & 119 & 1.8 & 3.6 & 121 & 1.8 & 3.6 \\
\hline 103 & 98 & 1.6 & 3.3 & 57 & 1.6 & 3.3 & 90 & 1.8 & 3.6 & 84 & 1.7 & 3.4 \\
\hline
\end{tabular}

shape, $S_{\mathrm{XRD}}$, were compared in order to evaluate the degree of agglomeration among crystallites. For the comparison the agglomeration factor, $1 / F$, is defined as (4). The surface area obtained from XRD, $S_{\mathrm{XRD}}$, was calculated by means of Eq. (5) where $\rho$ is the density of $\mathrm{ZnO}$, taken as $5.673 \mathrm{~g} \mathrm{~cm}^{-3}$, and $S_{\text {XRD }}$ is expressed in $\mathrm{m}^{2} \mathrm{~g}^{-1}$. The volume-weighted mean height $\left\langle H_{v}\right\rangle$ is equal to the apparent size for cylindrical crystallites from the (001) reflection and the equivalent diameter $\left\langle D_{v}\right\rangle$ is given by Eq. (6),

$$
\begin{gathered}
1 / F=\mathrm{S}_{\mathrm{BET}} / S_{\mathrm{XRD}}, \\
S_{\mathrm{XRD}}=\left(4\left\langle H_{v}\right\rangle+2\left\langle D_{v}\right\rangle\right) \times 10^{3} /\left\langle H_{v}\right\rangle\left\langle D_{v}\right\rangle \rho, \\
\left\langle D_{V}\right\rangle=\frac{3 \pi}{8} \epsilon_{h k 0} .
\end{gathered}
$$

The calculated values $S_{\mathrm{XRD}}, 1 / F$, and the obtained values $S_{\mathrm{BET}}$ are listed in Table I.

\section{RESULTS AND DISCUSSIONS}

The method used to obtain zinc oxide produces different particle shapes as a function of the precursor precipitation time. Among the shapes are porous spherical aggregates, obtained within the period of time from first turbidity until 75 min of precipitation. At longer periods of precipitation they are transformed to spherulitic needle aggregates and single acicular particles. SEM micrographs were published elsewhere (Sigoli et al., 1997). This morphological evolution is in agreement with thermodynamic principles and consequently with the natural occurrence of the precursor (Ghose, 1964). Transmission electron microscopy (Figure 3) revealed that the spherulitic aggregates are formed by acicular particles that consist of small crystallites [Figure 3(a)]. Although there are many terms for particle size and shape (Matyi et al., 1987), by considering these crystallites as cylindrical or spherical particles, the definitions of the Feret's diameter, maximum caliper diameter, equivalent circle, and equivalent sphere can be used to infer their diameter or size. From this consideration, the crystallites are approximately $15 \mathrm{~nm}$ across, appearing roughly isotropic [Figures 3(b) and 3(c)]. It is interesting to note the remarkable linear organization of these subparticles at the surface of a pseudomorph. This result is an indication of a crystallographic orientation relationship between the oxide and its precursor crystals. This relationship is a consequence of a topotactic transformation process. These results are similar to those observed by Bolis et al. (1989) and Auffrédic et al. (1995). The diameters of the crystallites seen in Figures 3(b) and 3(c) are close to the apparent crystallite sizes calculated in the direction perpendicular to the (100) plane (Table II).

The morphology was also evaluated by X-ray diffracto-

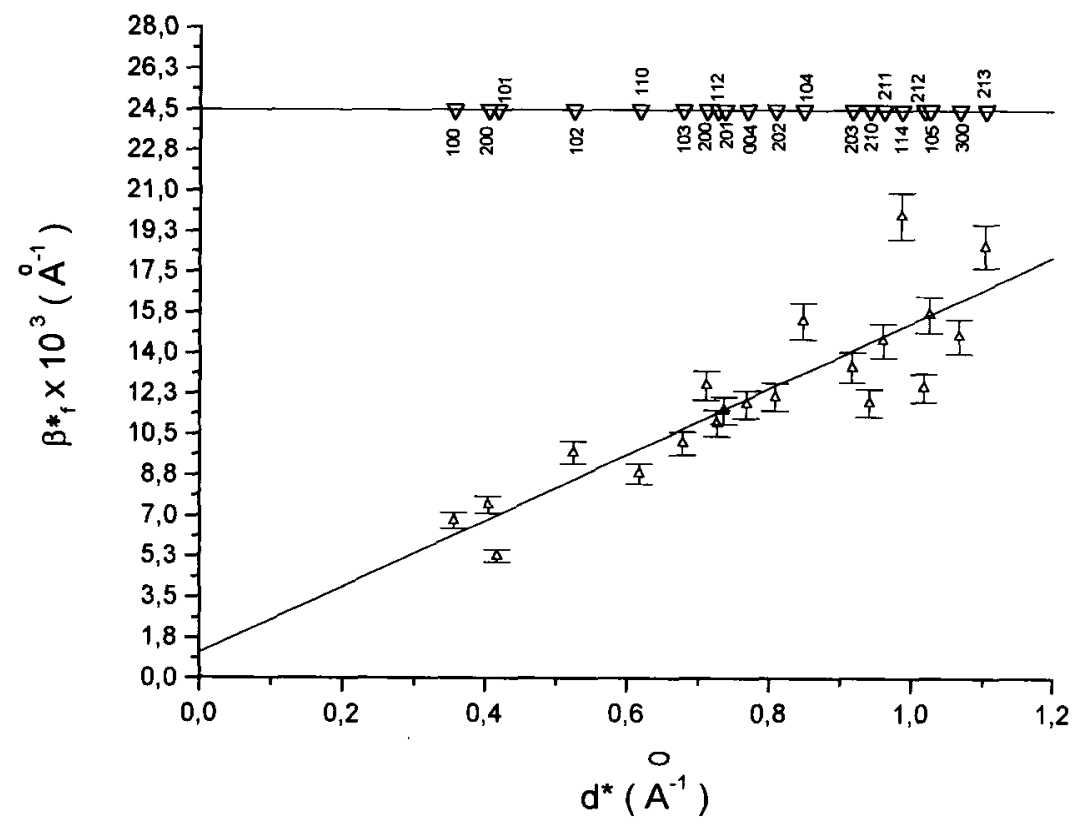

Figure 4. Indexed Williamson-Hall plot ( $\beta_{f}^{*}$ vs $d^{*}$ ) of $\mathrm{ZnO}-30$ obtained by thermal decomposition of precursor precipitated at $30 \mathrm{~min}$. 


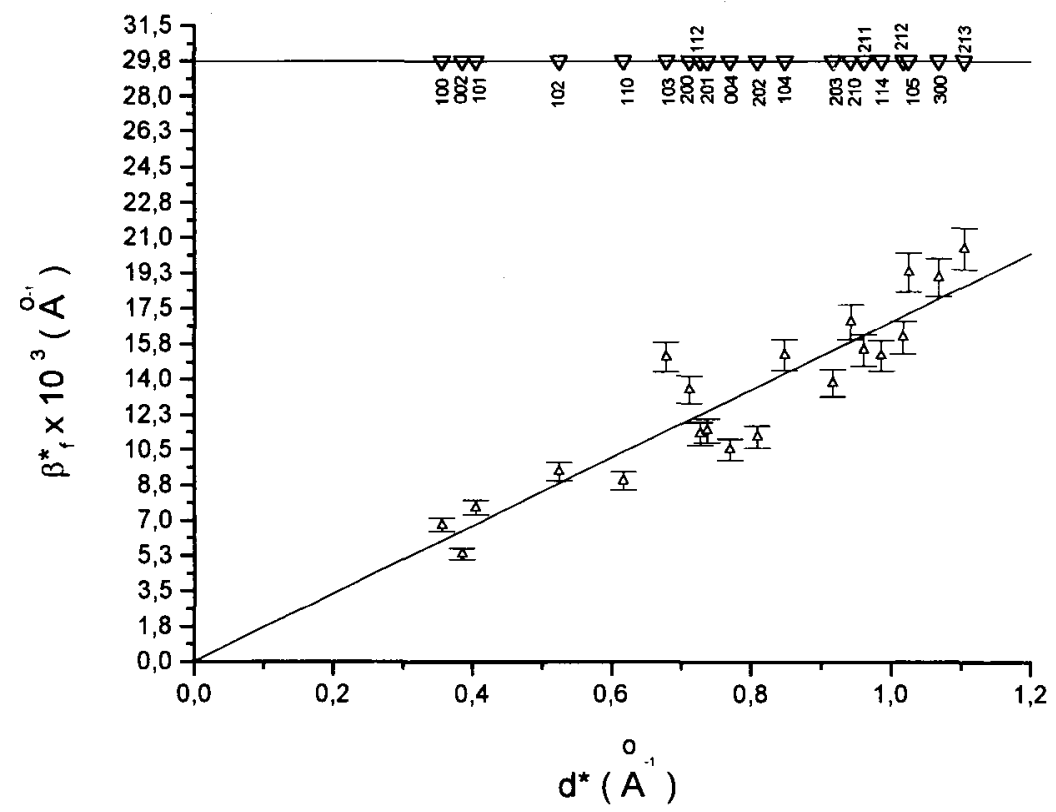

Figure 5. Indexed Williamson-Hall plot ( $\beta_{f}^{*}$ vs $d^{*}$ ) of $\mathrm{ZnO}-180$ obtained by thermal decomposition of precursor precipitated at $180 \mathrm{~min}$.

metric analysis. Zinc oxide samples obtained by thermal decomposition are crystalline and their X-ray diffractograms are characteristic of zincite (Figure 1). The intensity of the reflection (002) is substantially increased as a function of precursor precipitation time. However, from data in Table II crystallite size does not increase for the (002) indicating that the reflection intensity increase is related to preferred orientation in the samples. Moreover, the intensity increase of the reflection (002) is correlated to the number of isolated acicular particles or spherulitic needle aggregates in the samples. These shapes depend on precursor precipitation time as verified from electron scanning microscopy by Sigoli et al. (1997). The apparent crystallite size $\left(\epsilon_{h k l}\right)$ in the direction perpendicular to the $(002)$ plane is larger than for (100) (Table II), and consequently the crystallite shape may be regarded as almost cylindrical. These data are in agreement with the transmission electron microscopy results. From the Williamson-Hall plots (Langford et al., 1993) (Figures 4-7) it is immediately apparent that there is a scatter in the values of $\beta_{f}^{*}$ suggesting the utility of the single-line method to distinguish size and strain. There is a positive slope, and $\beta_{f}^{*}$ lines for $(h 00)$ and $(00 l)$ reflections have nonzero and slightly different slopes (plots not shown), indicative of the presence of microstrain. In this case, according to the rms strain calculated from the single-line method (Table II), all $\mathrm{ZnO}$ samples have a similar degree of microstrain, independent of the precursor precipitation time (Table II). For zinc oxide samples the agglomeration factor $(1 / F)$ was calculated from the data shown in Table I. The constant results indicate that the agglomeration degree between crystallites does not depend on precursor precipitation time.

In recent work, (Sigoli et al., 1999) it was observed that the zinc oxide excitation spectra present different bands as a function of particle shapes. Excitation spectra showed three bands at $377,405,430 \mathrm{~nm}$ for porous spherical aggregates

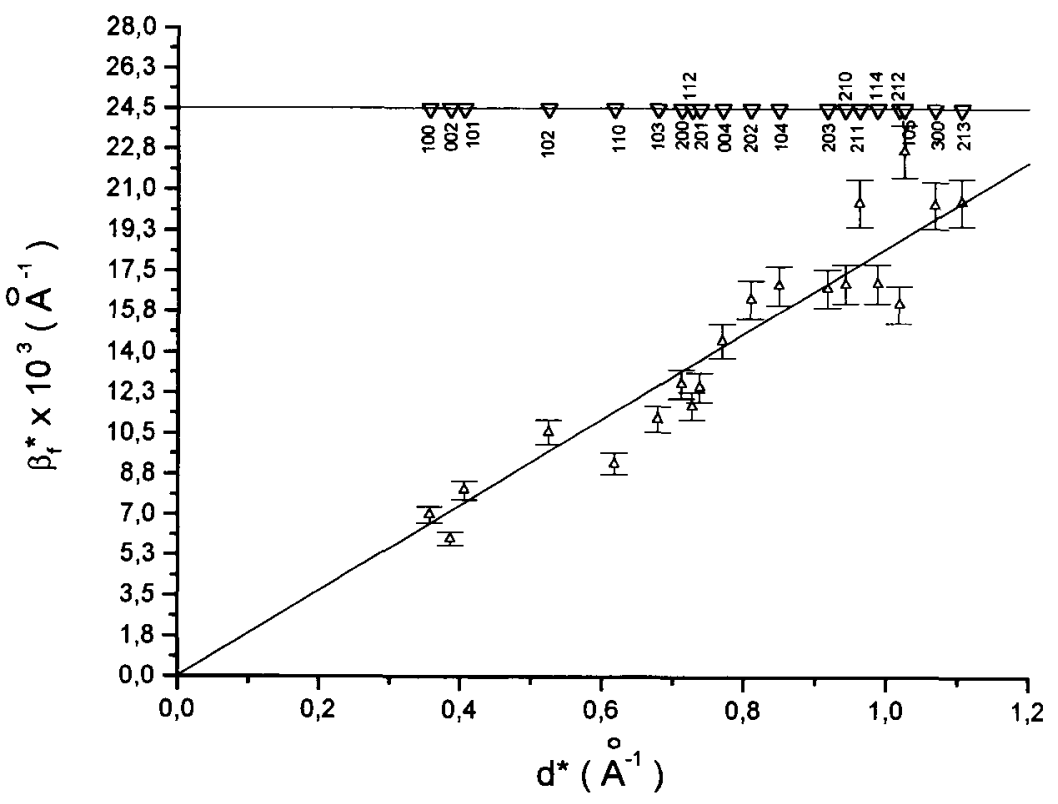

Figure 6. Indexed Williamson-Hall plot ( $\beta_{f}^{*}$ vs $d^{*}$ ) of $\mathrm{ZnO}-250$ obtained by thermal decomposition of precursor precipitated at $250 \mathrm{~min}$. 


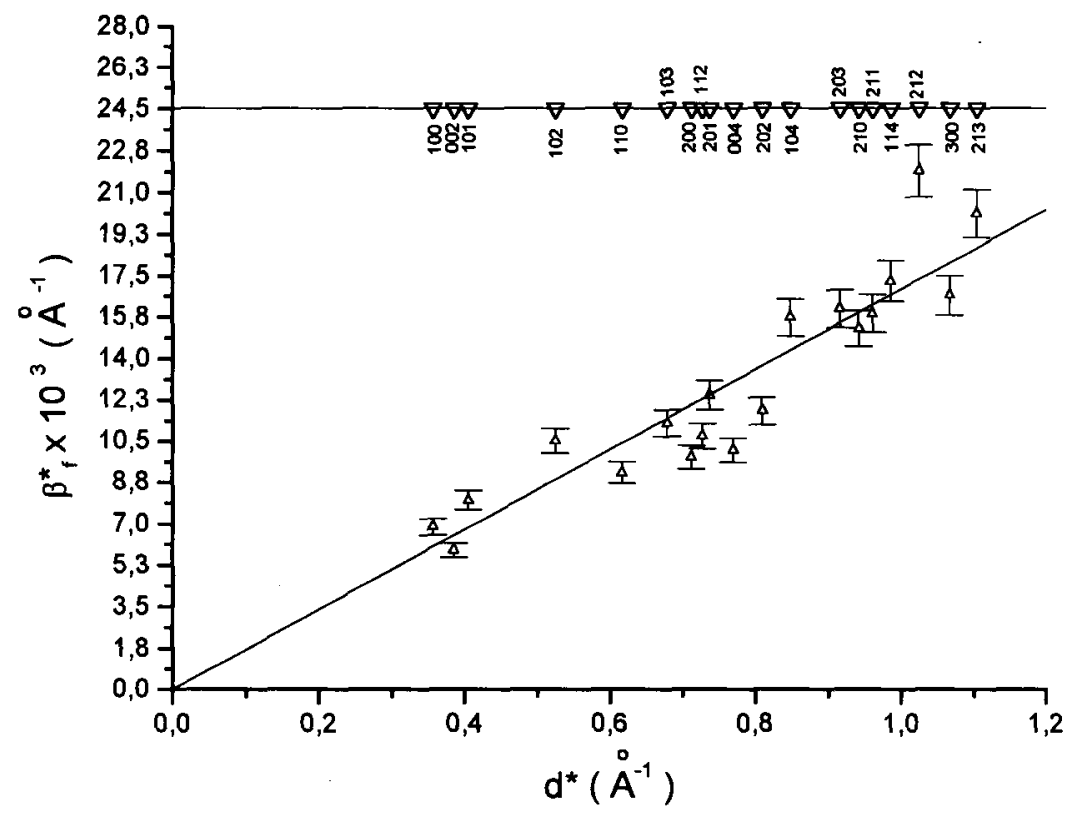

Figure 7. Indexed Williamson-Hall plot ( $\beta_{f}^{*}$ vs $d^{*}$ ) of $\mathrm{ZnO}-440$ obtained by thermal decomposition of precursor precipitated at $440 \mathrm{~min}$.

and only a well-defined band at $377 \mathrm{~nm}$ for spheruliticshaped particles and needle particles. These different bands were correlated with: (i) the morphological changes, (ii) the urea decomposition during precursor precipitation process, (iii) the thermal decomposition of the precursor, and (iv) with lattice defects in the zinc oxide structure. The present results allow us to infer that the different excitation bands verified before (Sigoli et al., 1999) can be attributed to electronic defects originating from either particle shape or from oxygenated groups present on $\mathrm{ZnO}$ surface instead of lattice defects, which are invariable as a function of precursor precipitation time.

\section{CONCLUSIONS}

From the results it is possible to conclude that the precursor precipitation period directly influences the zinc oxide morphology but influences neither the average crystallite size nor the microstrain for $\mathrm{ZnO}$ samples obtained by this route. It is shown that the spherulitic aggregates are formed by needlelike particles, which are built of anisotropic crystallites. All the zinc oxide samples presented microstrain that is qualitatively shown by Williamson-Hall plots. The presence of microstrain in the samples is likely a consequence of the low temperature and short period of time used for precursor thermal decomposition. The results presented in this work also allow us to infer that the different excitation bands verified by Sigoli et al. (1999), can be attributed to electronic defects originating from the particle shape or from oxygenated groups present on the $\mathrm{ZnO}$ surface instead of lattice defects.

Therefore, using this route, it is possible to prepare zinc oxide with different morphologies without microstructural alterations. Such preparation may be very important in affecting several properties of $\mathrm{ZnO}$ that may be useful in the manufacturing of optical devices.

\section{ACKNOWLEDGMENTS}

Financial support by $\mathrm{CNPq}$ and FAPESP is gratefully acknowledged. F.A.S. thanks CNPq for a scholarship. The authors would like to thank Professor Maria do Carmo Gonçalves from UNICAMP for her assistance in TEM analysis.

Auffrédic, J. P., Boultif, A., Langford, J. I., and Louër, D. (1995). "Early stages of crystallite growth of $\mathrm{ZnO}$ obtained from an oxalate precursor," J. Am. Ceram. Soc. 78, 323-328.

Blasse, G., and Grabmaier, B. C. (1994). Luminescent Materials (Springer, Berlin), pp. 60-64.

Bolis, V., Fubini, B., Giamello, E., and Reller, A. (1989). "Effect of form on the surface reactivity of differently prepared zinc-oxides," J. Chem. Soc., Faraday Trans. $185,855$.

Castellano, M. A., and Matijévic, E. (1989), "Uniform colloidal zinc compounds of various morphologies," Chem. Mater. 1, 78-82.

Collins, I. R., and Taylor, E. S. (1992). "Nonaqueous thermaldecomposition route to colloidal inorganic oxides," J. Mater. Chem. 2 , $1277-1281$

Georgobiani, A. N., Butkhuzi, T. V., Zadauly, E., Kekelidze, N. P., and Khulordava, T. G. (1993). "Optical-properties of zinc oxide dielectric layers," Inorg. Mater. (Transl. of Neorg. Mater.) 29, 1249-1252.

Ghose, S. (1964). "The crystal structure of hydrozincite, $\mathrm{Zn}_{5}(\mathrm{OH})_{6}$ $(\mathrm{CO} 3)_{2}$, , Acta Crystallogr. 17, 1051-1057.

Haruta, M., and Delmon, B. (1986). "Preparation of homodisperse solids," J. Chim. Phys. Phys.-Chim. Biol. 83, 859-868.

Koudelka, L., and Horák, J. (1994). "Morphology of polycrystalline ZnO and its physical-properties," J. Mater. Sci. 29, 1497-1500.

Langford, J. I. (1992). "The use of the Voigt function in determining microstructural properties from diffraction data by means of pattern decomposition," in Accuracy in Powder Diffraction II, edited by E. Prince and J. K. Stalik (NIST Special Publication No. 846, Gaithersburg), pp. 110126.

Langford, J. I., Boultif, A., Auffrédic, J. P., and Louer, D. (1993). "The use of pattern decomposition to study the combined X-ray diffraction effects of crystallite size and stacking faults in ex-oxalate zinc oxide," J. Appl. Crystallogr. 26, 22-33.

Langford, J. I., Delhez, R., Keijser, Th. H., and Mittemeijer, E. J. (1988). "Profile analysis for microcrystalline properties by the Fourrier and other methods," Aust. J. Phys. 41, 173-187.

Louër, D., Auffrédic, J. P., Langford, J. I., Ciosmak, D., and Niepce, J. C. (1983). "A precise determination of the shape, size and distribution of crystallites in zine oxide by X-ray line-broadening analysis," J. Appl. Crystallogr. 16, 183-191.

Matijévic, E. (1985). "Production of monodispersed colloidal particles," Annu. Rev. Mater. Sci. 15, 483-516.

Matyi, R. J., Schwartz, L. H., and Butt, J. B. (1987). "Particle size, particle size distribution and related measurements of supported metal catalysts," Catal. Rev. Sci. Eng. 29, 41-99. 
Sigoli, F. A., Davolos, M. R., and Jafelicci, Jr., M. (1997). "Morphological evolution of zinc oxide originating from zinc hydroxide carbonate," J. Alloys Compd. 262-263, 292-295.

Sigoli, F. A., Davolos, M. R., and Jafelicci, Jr., M., (1999). "Morphological control and luminescence properties of zinc oxide," Adv. Sci. Technol. (Faenza, Italy) 27, 45-52.

Toraya, H. (1990). "'Operating manuals for computer programs: PRO-FIT and WPPF."
Vanheusden, K., Seager, C. H., Warren, W. L., Tallant, D. R., Caruso, J., Hampden-Smith, M. J., and Kodas, T. J. (1997). "Green photoluminescence efficiency and free-carrier density in $\mathrm{ZnO}$ phosphor powders prepared by spray pyrolysis," J. Lumin. 75, 11-16.

Zampronio, C. G., Davolos, M. R., Stucchi, E. B., and Jaffelicci, Jr., M. (1995). "Spherical particles of pure and manganese doped zinc oxide and zinc hydroxicarbonate," Mater. Res. Soc. Symp. Proc. 372, 69-74. 